\title{
Algorithm to Estimate Direction of Arrival with Interpolated Array Elements for Coprime Array Holes
}

\author{
Guicai Yu \\ Physical Science and Engineering Technology School, Yichun University, Yichun 336000, China \\ Correspondence should be addressed to Guicai Yu; guicai.yu@gmail.com
}

Received 13 July 2020; Accepted 17 August 2020; Published 31 August 2020

Guest Editor: Wang Zheng

Copyright (C) 2020 Guicai Yu. This is an open access article distributed under the Creative Commons Attribution License, which permits unrestricted use, distribution, and reproduction in any medium, provided the original work is properly cited.

\begin{abstract}
A novel method for adding antennas in the coprime arrays is introduced in this study, in order to solve the problem of the reduced degree of freedom of the array in the hole-existing coprime arrays. The minimum number of antennas interpolated in the algorithm maximizes the available degrees of freedom of virtual arrays, and the number of interpolated antennas does not change the original aperture size of the coprime arrays. With the proposed algorithm, the estimate of the direction of arrival is more accurate for a given signal-to-noise ratio. The scheme first finds the regular pattern of hole positions in virtual array elements, and then, according to the regular pattern, the position of the hole of the partial virtual array element is interpolated with the array element antenna at the position of the corresponding coprime arrays. The holes of the virtual array element are filled, giving virtual uniform continuous array elements with maximum degrees of freedom. We use the ESPRIT, and the simulation results show that the proposed algorithm improves the accuracy and resolution of estimates of the direction of arrival.
\end{abstract}

\section{Introduction}

Estimating the direction of arrival (DOA) is an important research topic in the field of array signal processing and is widely applied in radar, communications, electronic countermeasures, and many other fields [1-7]. Although estimating the DOA can be well addressed by using traditional uniform linear arrays (ULAs), such estimates may be accurate when the number of signal sources exceeds the number of sensors because of constraints imposed on the degrees of freedom by the number of sensors in the array.

Coprime arrays [8-11] are coming to the fore in academia because provide accurate DOA estimation when signal numbers are greater than the number of array elements. Moreover, virtual arrays derived from a coprime array contain more virtual sensors, thereby allowing for more accurate DOA estimates. Virtual arrays thus derived contain both uniform continuous parts and discrete parts. In 2011, Pal and Vaidyanathan used the continuous part of virtual arrays to estimate DOA by harnessing spatial smoothing algorithms [12] following which several related DOA estimation algorithms were developed [13-15].
However, these algorithms did not exploit discrete sensors, resulting in inefficient use of apertures of virtual arrays and reduction in the degree of freedom of the coprime array.

An increased number of virtual sensors must be used to avoid reduction the degree of freedom. Although the sparse signal reconstruction algorithm [16] leverages all virtual sensors, it incurs a high computational cost because it significantly reuses equivalent virtual signals obtained by covariance vectorization of the sample. Boudaher et al. [17] proposed to apply temporal signal coherence to fill partial holes in moving coprime arrays, but precise temporal correlation is difficult to realize in actual applications. In addition, Zhang et al. [18] used sensors around the holes of virtual sensors to fit the virtual array response of the missing position. However, the significant error in the fitted virtual array response due to the presence of continuous holes reduced the accuracy of such estimates.

To resolve these issues, we propose herein an improved approach that is based on the discovery of a regular pattern in the position of the hole in a virtual array element, which allows the interpolation of a small number of array-element antennas at the position of coprime 
arrays to fill these holes and thereby maximize the degree of freedom of virtual array elements. The results of a simulation and a comparison with the primary parameters from previous work $[15,18]$, including DOA estimates, estimated resolution, and estimated accuracy, clearly show the advantages of the proposed method.

\section{Mathematical Model}

The coprime array consists of two groups of a uniform linear array. As shown in Figure 1, the number of physical array elements in the first group is $N$, the distance between array elements is $M d$, the number of physical array elements in the second group is $2 M$, and the corresponding distance between array element is $N d$. Given that the first array element is shared, the total number of array elements is $2 M+N-1$. Furthermore, $P=\left[P_{1}, P_{2}, \ldots, P_{(2 M+N-1)}\right]$ indicates the position of the physical array elements in the coprime array. Note that $M<N$, so $d$ is the half wavelength of the signal when arrays are coprime with each other.

For simplicity, we assume $K$ uncorrelated signals with different incident angles $\theta=\left[\theta_{1}, \theta_{2}, \ldots, \theta_{K}\right]$ with respect to the receiver array. The actual received data at the $t$ snapshot are

$$
X(t)=A(\theta) s(t)+n(t),
$$

where $A(\theta)=\left[a\left(\theta_{1}\right), a\left(\theta_{2}\right), \ldots, a\left(\theta_{K}\right)\right]$ is the array manifold of dimension $(2 M+N-1) K$, and element $k$ is

$$
\begin{aligned}
a\left(\theta_{k}\right) & =\left[e^{-j(2 \pi / \lambda) P_{1} \sin \left(\theta_{k}\right)}, \ldots, e^{-j(2 \pi / \lambda) P_{(2 M+N-1)} \sin \left(\theta_{k}\right)}\right], \\
X(t) & =\left[x_{P 1}(t), x_{P 2}(t), \ldots, x_{P(2 M+N-1)}(t)\right]^{T},
\end{aligned}
$$

is the received-signal vector. The direction vector $a\left(\theta_{k}\right)=$ $\left[e^{(-j 2 \pi / \lambda) P_{1} \sin \left(\theta_{k}\right)}, \ldots, e^{-j(-j 2 \pi / \lambda) P_{(2 M+N-1)} \sin \left(\theta_{k}\right)}\right]$, and $n(t)$ is the additive white Gaussian noise vector with power $\sigma_{n}^{2}$ and is independent between signals. The theoretical covariance matrix of the received signal $x(t)$ in the coprime array is

$$
R_{x x}=E\left[x(t) x^{H}(t)\right]=A(\theta) \sum A^{H}(\theta)+\sigma_{n}^{2} I,
$$

where $E(\cdot)$ is the expectation, $(\cdot)^{H}$ is the conjugate transposition,

$$
\Sigma=E\left[s(t) s^{H}(t)\right]=\operatorname{diag}\left(\left[\sigma_{1}^{2}, \sigma_{2}^{2}, \ldots, \sigma_{K}^{2}\right]\right),
$$

is a diagonal matrix of dimension $K \times K, \sigma^{2}=\left[\sigma_{1}^{2}, \sigma_{2}^{2}, \ldots\right.$, $\left.\sigma_{K}^{2}\right]^{T}$ is the power vector of the signal, $I$ is the unit matrix of dimension $(2 M+N-1)(2 M+N-1)$, and the theoretical covariance matrix $R_{x x}$ is acquired during a single snapshot. In general, the adopted sampled covariance matrix is obtained via the samples at snapshot $T$ :

$$
\bar{R}_{x x}=\frac{1}{T} \sum_{t=1}^{T} x(t) x^{H}(t) .
$$

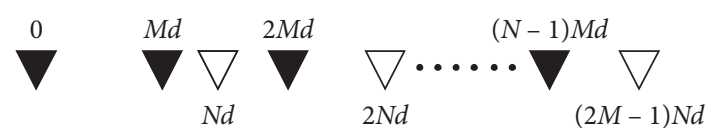

FIgURe 1: Model of array.

\section{DOA Estimation Algorithm Interpolated in the Hole of Virtual Array Element}

3.1. Construction of Virtual Array. The virtual array is constructed from coprime arrays. In particular, equivalent virtual signals are obtained from the vectorization of the sample covariance of received signals in the coprime arrays. Explicitly, the position of virtual elements is given as

$$
S=\left\{P_{i}-P_{j} \mid i, j=1,2, \ldots, 2 M+N-1\right\} .
$$

To define the position $P$ in the coprime array, $S$ can be denoted by the union of three subsets:

$$
\begin{aligned}
& S=S_{1} \cup S_{2} \cup S_{3}, \\
& S_{1}=\left\{M n_{i}-M n_{j}, 0 \leq n_{i}, n_{j} \leq N-1\right\}, \\
& S_{2}=\left\{N m_{i}-N m_{j}, 0 \leq m_{i}, m_{j} \leq 2 M-1\right\}, \\
& S_{3}=\left\{\begin{array}{rr} 
\pm(N m-M n), & 0 \leq m \leq 2 M-1, \\
0 \leq n \leq N-1 .
\end{array}\right\},
\end{aligned}
$$

Analysis of the above equations shows that equations (8) and (9) are the set of self-differences corresponding to the received signal, and equation (10) is the set of mutual differences corresponding to the received signal, where $S_{1}$ and $S_{2}$ are subsets of $S_{3}$, so the position of the virtual array element can be represented as

$$
S=\{ \pm(n M d-N m d)\}, \quad 0 \leq n \leq N-1,0 \leq m \leq 2 M-1 .
$$

3.2. Array Element Interpolated in Coprime Array. Figure 2 shows the position of the coprime array and the virtual array when $M=3$ and $N=5$.

The virtual array is composed of two parts, namely, the continuous array and the discrete array. According to the position of the virtual elements in equation (11), the continuous array spans from $-(M N+N-1)$ to $M N+M-1$, which is equivalent to the range $-17 d$ to $17 d$, as shown in Figure 2(b). The position of the hole of the discrete array is $P_{0}= \pm(M N+a M+b N)$, with $P_{0}<(2 M-1) N$, where $a \geq 1, b \geq 0$. This is equivalent to $\{ \pm 18 d, \pm 21 d, \pm 23 d$, $\pm 24 d\}$, as shown in Figure 2(b). Given the pattern of the hole positions, we infer that all holes may be filled by interpolating the array element antenna at the position of the maximum continuous hole corresponding to the coprime array. In Figure 2(b), the maximum continuous position is $23 d$ and $24 d$. Two antennas were added at $23 d$ and $24 d$ in 


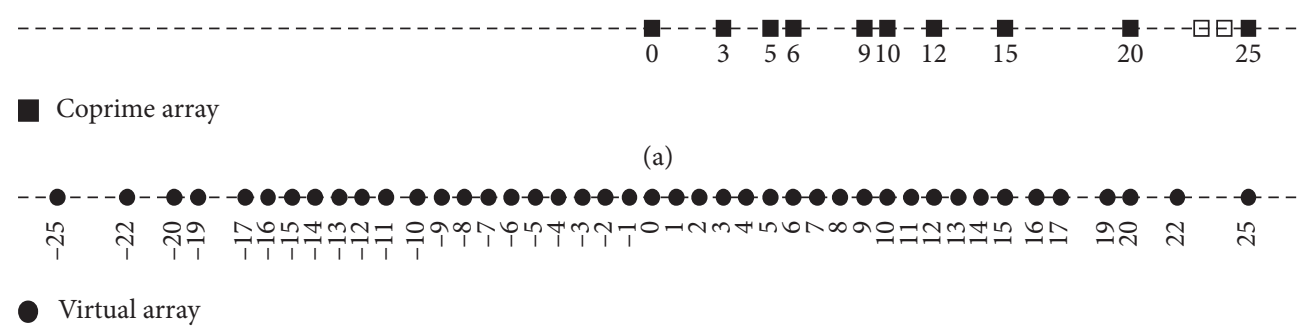

(b)

Figure 2: (a) Coprime array (b) virtual array.

the coprime arrays, so we obtain the positions $\pm 23 d$ and $\pm 24 d$ for holes based on the difference between the antenna positions at $0 d$ in arrays and the positions of the two added antennas. The positions $\pm 18 d$ of holes are obtained from the difference between the position $24 d$ of the added antenna and the position $6 d$ in the arrays. The positions $\pm 21 d$ of holes are obtained from the difference between the position $24 d$ of the added antenna and the position $3 d$ in the arrays. Consequently, all holes are filled, so the virtual arrays are continuous from $-25 d$ to $25 d$. In addition, because all newly added elements are located within the original aperture, the degree of freedom of the arrays may be increased without expanding the original array aperture.

3.3. DOA Estimate Based on ESPRIT Algorithm. After adding new elements to $A(\theta)$ in equation (1), vectorizing the sampled covariance of the received signals gives

$$
z=\operatorname{vec}\left(R_{x x}\right)=B \sigma^{2}+\sigma_{n}^{2} i,
$$

where $i=\operatorname{vec}(I), B=\left[a^{*}\left(\theta_{1}\right) \otimes a\left(\theta_{1}\right), \ldots, a^{*}\left(\theta_{K}\right) \otimes a\left(\theta_{K}\right)\right]$, the element in $a^{*}\left(\theta_{k}\right) \otimes a\left(\theta_{k}\right)$ can be expressed as $e^{-j(2 \pi / \lambda)\left(P_{i}-P_{j}\right) \sin \left(\theta_{k}\right)}$, and $p_{i}$ and $p_{j}$ represent antennas $i$ and $j$ in the coprime arrays that generated the equivalent virtual signal for each virtual array element in the virtual array. Liu and Vaidyanathan [19] suggested that the equivalent covariance of virtual array signals can be acquired by rearranging the elements in $z$ in the Toeplitz matrix configuration. Given the symmetry about position 0 between the position of the array elements $-p$ and $p$, the elements of their equivalent virtual signals in $B$ are conjugate to each other. Thus, only the equivalent virtual signal must be processed for the continuous part at positions from 0 to $p$ in the virtual array, as shown in Figure 3.

The covariance of the virtual array is denoted as

$$
R_{z z}=T(y)=\left[\begin{array}{cccc}
z_{(0)} & z_{(1)}^{*} & \cdots & z_{(25)}^{*} \\
z_{(1)} & z_{(0)} & \cdots & z_{(24)}^{*} \\
\vdots & \vdots & \ddots & \vdots \\
z_{(25)} & z_{(24)} & \cdots & z_{(0)}
\end{array}\right],
$$

where $T(y)$ is the Hermitian Toeplitz matrix, and in the first row, $z_{(i)}$ means the equivalent virtual signal at array element $i$ in the virtual array.

In the uniform linear array of Figure 3, the virtual subarray $S_{Y}$ is assembled from virtual sensors at positions

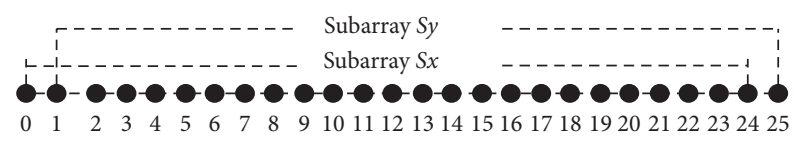

Figure 3: Subarray of virtual array.

from $1 d$ to $25 d$, where $d$ is the corresponding virtual sensor displacement in each subarray.

The signal received by the virtual subarray at time $t$ can be expressed as

$$
\begin{aligned}
& S_{X}(t)=A_{x} s(t)+n_{X}(t), \\
& S_{Y}(t)=A_{Y} s(t)+n_{Y}(t),
\end{aligned}
$$

where $A_{X}$ and $A_{Y}$ are the steering matrices of the virtual subarrays $S_{X}$ and $S_{Y}$ and $n_{X}$ and $n_{Y}$ are the additive white noise in the virtual subarrays.

The virtual subarrays $S_{X}$ and $S_{Y}$ are displaced a distance $d$ in a uniform linear array, indicating that subarrays $S_{X}$ and $S_{Y}$ are translation invariant. The received signal $S_{Y}(t)$ from subarray $S_{Y}$ in equation (15) can be expressed by using the steering matrix of the subarray $S_{X}$ as

$$
S_{Y}(t)=A_{X} \Phi s(t)+n_{Y}(t),
$$

where

$$
\Phi=\operatorname{diag}\left\{e^{-j 2 \pi / \lambda d \sin \left(\theta_{1}\right)}, \ldots, e^{-j 2 \pi / \lambda d \sin \left(\theta_{K}\right)}\right\}
$$

is the diagonal matrix of dimension $K \times K$, also called the rotation operator. The theoretical covariances of the virtual subarrays $S_{X}(t)$ and $S_{Y}(t)$ are

$$
\begin{aligned}
& R_{S_{X} S_{X}}=A_{X} \Sigma A_{X}^{H}+\sigma_{n}^{2} I, \\
& R_{S_{Y} S_{Y}}=A_{X} \Phi \Sigma \Phi^{H} A_{X}^{Y}+\sigma_{n}^{2} I .
\end{aligned}
$$

The rotation invariance of the virtual subarrays leads to the rotation invariance of the virtual subarray signals. Therefore, the ESPRIT algorithm can be used in the field of the coprime array. In the virtual array, the equivalent virtual signal is obtained by vectorizing the sample covariance of the signal received by the coprime array, whereas the theoretical covariance matrices $R_{S_{X} S_{X}}$ and $R_{S_{Y} S_{Y}}$ are the values at the single snapshot and are not available. 
Since the virtual subarray is on the virtual uniform linear array, the virtual signal subspace of the subarray is also contained in the signal subspace of the virtual array.

The virtual covariance matrix $R_{z z}$ in equation (13) can be decomposed into its eigenvalues as

$$
R_{z z}=E_{S} \Lambda_{S} E_{S}^{H}+E_{N} \Lambda_{N} E_{N}^{H}
$$

where $\Lambda_{S}$ is the diagonal matrix with a maximum of $K$ eigenvalues on the diagonal, is the subspace of the signal composed of the corresponding eigenvectors, $\Lambda_{N}$ is the diagonal matrix consisting of relatively small eigenvalues, and $E_{N}$ is the noise subspace composed of the corresponding eigenvector. Based on the definition of the virtual subspace, the signal subspace $E_{S X}$ and $E_{S Y}$ corresponding to subarrays $S_{X}$ and $S_{Y}$ can be obtained by replacing the first and last rows of $E_{s}$, respectively, with

$$
E_{S}=\left[\begin{array}{c}
E_{S}(1,:) \\
E_{S X}
\end{array}\right]=\left[\begin{array}{c}
E_{S Y} \\
E_{S}(\text { end, : })
\end{array}\right] .
$$

The orthogonality between signal and noise subspaces means that a unique nonsingular matrix $T$ of dimension $K \times$ $K$ should exist with

$$
\begin{aligned}
& E_{S X}=A_{X} T, \\
& E_{S Y}=A_{Y} T=A_{X} \Phi T,
\end{aligned}
$$

where the definition of the matrix $E_{S X Y}=\left[E_{S X} E_{S Y}\right]$ and the rank of the matrix $E_{S X Y}$ should be $K$ because the same subspace is spanned by $E_{S X}$ and $E_{S Y}$. Meanwhile, a fullranked matrix $Q=\left[Q_{X} ; Q_{Y}\right]$ with dimension $2 K \times K$ should exist and be orthogonal to $E_{S X Y}$ :

$$
E_{S X Y} Q=E_{S X} Q_{X}+E_{S Y} Q_{Y}=0 .
$$

Combining equation (24) with equations (22) and (23) gives

$$
-A_{X} T Q_{X} Q_{Y}^{-1}=A_{X} \Phi T .
$$

Using the definition $F=-Q_{X} Q_{Y}^{-1}$, the rotation-invariance between signal subspace of subarrays $E_{S X}$ and $E_{S Y}$ is

$$
E_{S Y}=E_{S X} F \text {. }
$$

The rotation operator $F$ between signal subspaces is

$$
F=E_{S X}^{+} E_{S Y},
$$

where $E_{S X}^{+}$is the pseudoinverse of $E_{S X}$. Substituting $F$ into equation (25)fd26 gives

$$
A_{X} T F=A_{X} \Phi T
$$

where $T$ is invertible and $A_{X}$ is a full rank matrix. This gives

$$
\Phi=T F T^{-1}
$$

Therefore, $\Phi$ and $F$ are similar matrices with the same eigenvalues. Since the DOA of the incident source in equation (17) is included in $\Phi$, the estimate of $K$ DOAs can be expressed as

$$
\overline{\theta_{k}}=\arcsin \left[-\frac{1}{\pi} \operatorname{Im}\left(\operatorname{In} F_{k}\right)\right],
$$

where $F_{k}$ is eigenvalue $k$ of $F$ and $\operatorname{Im}(\cdot)$ gives the imaginary part.

3.4. Algorithm Steps. The steps of the proposed algorithm are as follows:

Step 1: after adding the antennas, calculate the sample covariance $R_{x x}$ of the coprime array and vectorize it as per equation (12)

Step 2: compute the covariance $R_{z z}$ of the virtual array in equation (13)

Step 3: obtain the corresponding signal subspace $E_{S X}$ and $E_{S Y}$ of the virtual arrays $S_{X}$ and $S_{Y}$ in equation (21)

Step 4: calculate the rotation operator $F$ in equation (27) according to the rotational invariance of the signal subspace

Step 5: calculate the eigenvalues $F_{k}(k=1,2, \ldots, K)$ of the rotation operator $F$ and estimate the DOA by using equation (30)

\section{Stimulation Results}

The performance of the proposed algorithm is verified through a simulation. In the coprime array, $M=3, N=5$, and the array element positions are $[0 d, 3 d, 5 d, 6 d, 9 d$, $10 d, 12 d, 15 d, 20 d, 23 d, 24 d, 25 d$ ]. The results of the simulation results are compared with published results $[15,18]$. The classical DOA estimation algorithm of Zhou and Zhou [15] leverages the ESPRIT algorithm for signal processing of continuous virtual arrays with unexploited holes. The method proposed by Zhang et al. [18] fits the array response of holes and estimates the DOA by using spatial smoothing for the entire virtual array.

4.1. Experiment 1: Feasibility Analysis. Given the limitations in the degree of freedom of Zhou and Zhou [15], experiment 1 is compared with the results of Zhang et al [18]. We use 21 signal sources evenly distributed over the range $\left[-60^{\circ}, 60^{\circ}\right]$. Assuming that more signals are incident on the sensor than the number of sensors, the signal-to-noise ratio (SNR) is $\mathrm{SNR}=0$, and the number of snapshots is $T=500$.

Figure 4 shows the estimate of the DOA by the proposed method as a function of signal source. These results show that the DOA estimates are accurate for the 21 signal sources. Figure 5 shows the DOA estimated by using the algorithm of Zhang et al. [18], which shows that only 19 DOA estimates are detected.

To validate the increase in array freedom provided by the method proposed herein, we assume that 24 signal sources are evenly distributed over a range $\left[-60^{\circ}, 60^{\circ}\right]$, which is the same as used by Zhang et al. [18], The SNR is SNR = 10, and the snapshot number is $T=500$. As shown in Figure 6, 24 DOAs are detected, indicating an improvement in the array freedom over the 20 DOA detected by Zhang et al [18]. 


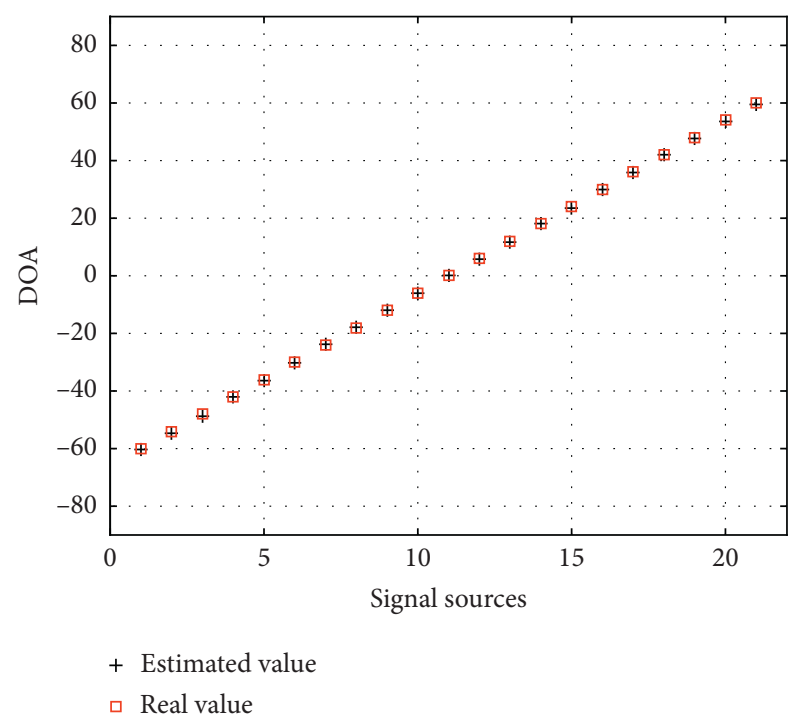

FIGURE 4: Estimate of DOA by using proposed method as a function of signal sources.

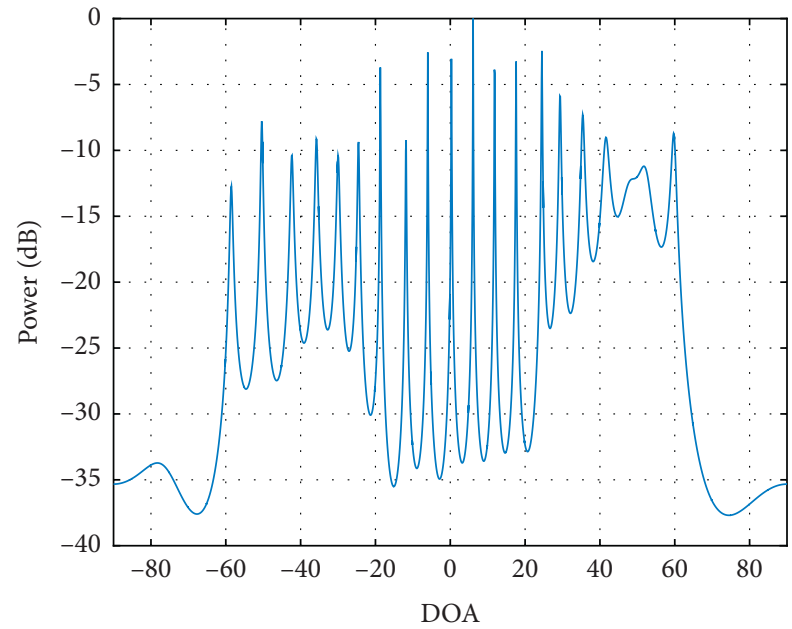

_ Algorithm proposed by Zhang et al. [18]

FIGURE 5: DOA estimation of the proposed method by Zhang et al. [18].

4.2. Experiment 2: Comparison of Estimation Accuracy. The root-mean-square error (RMSE) of the estimated angle was used to compare the estimation accuracy of DOA which is higher. The RMSE is defined as

$$
\mathrm{RMSE}=\sqrt{\frac{1}{K Q} \sum_{k=1}^{K} \sum_{q=1}^{Q}\left(\theta_{k, q}-\theta_{k}\right)^{2}},
$$

where $K$ is the number of signal sources, $Q$ is the number of MonteCarlo experiments, $\theta_{k, q}$ is the angle estimated for signal $k$ in the $q$ th MonteCarlo experiment, and $\theta_{k}$ is the $k$ th DOA. In experiment $2, Q=500$ and $K=13$, uniformly distributed over the range $\left[-60^{\circ}, 60^{\circ}\right]$.

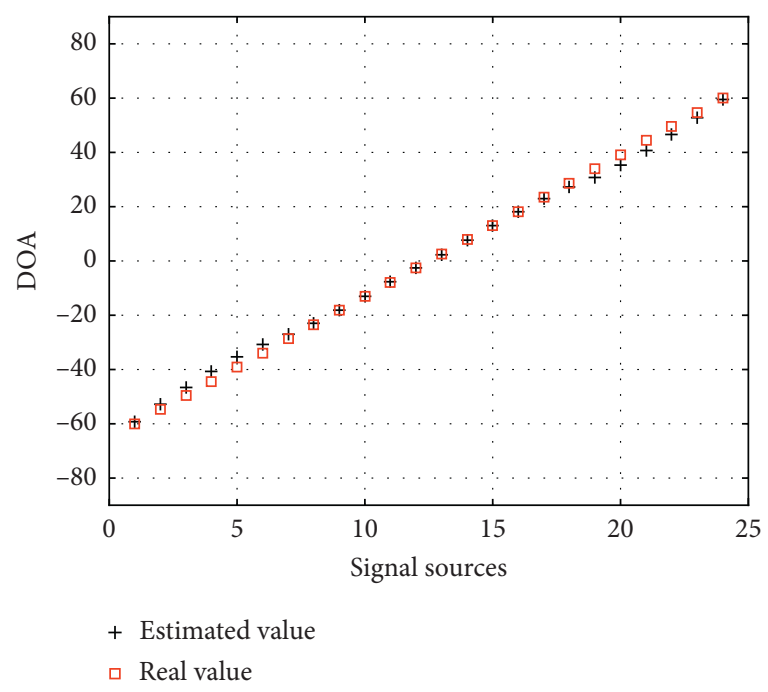

FIgURE 6: DOA estimate by proposed method with 24 signal sources.

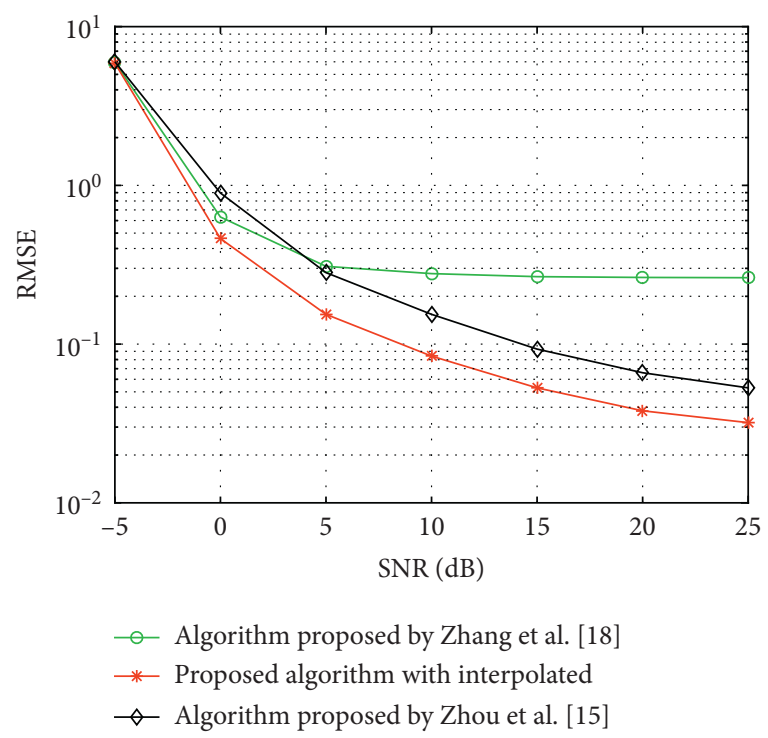

FIGURE 7: Estimation accuracy of the three algorithms as a function of signal-to-noise ratio.

Figure 7 shows the RMSE of the estimated angle estimation as a function of the SNR. The SNR ranges from -5 to $25 \mathrm{~dB}$ in $5 \mathrm{~dB}$ steps, and the number of snapshots is 500 . As can be seen in Figure 7, the RSME decreases with increasing SNR. Compared with the published algorithms $[15,18]$, the algorithm proposed herein produces a smaller RSME for the estimated angle and is thus more accurate than the published algorithms.

Figure 8 plots the RMSE of the angle estimate as a function of the number of snapshots, where SNR is taken to be $10 \mathrm{~dB}$. These results show that, compared with the published algorithms [15, 18], the algorithm proposed herein produces a smaller RMSE for estimating the angle. Such as, it is about 0.2 smaller than the algorithm in the 


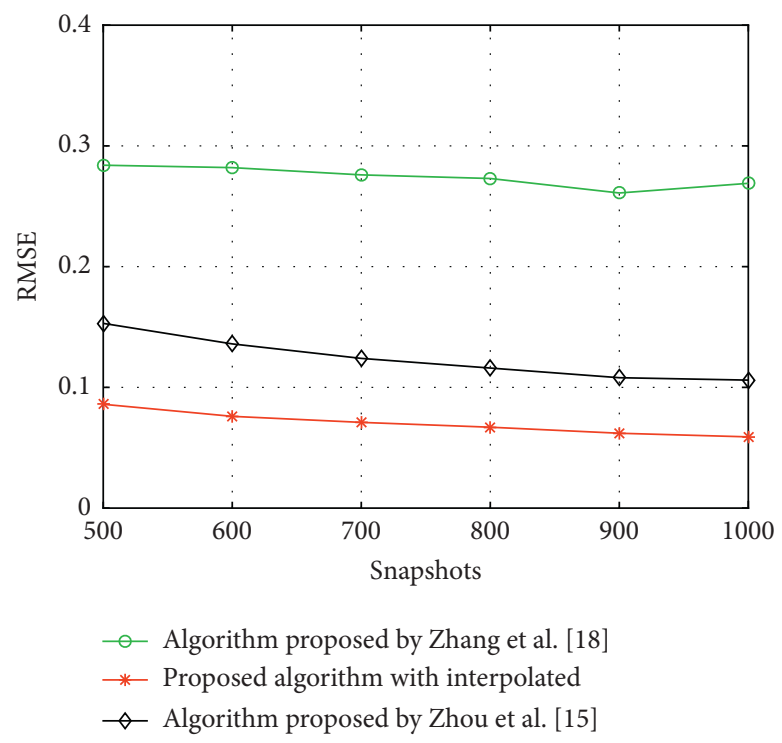

FIGURE 8: Estimation accuracy of the three algorithms as a function of number of snapshots.

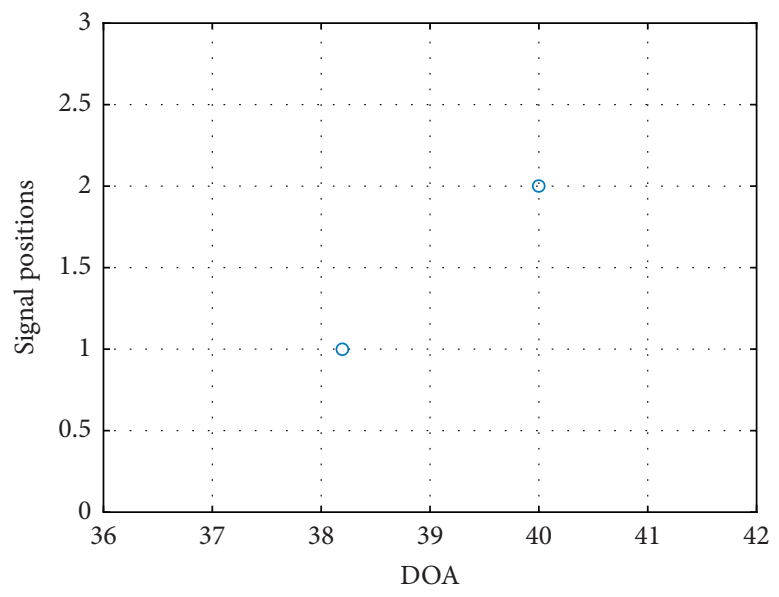

○ Proposed algorithm with interpolated

FIGURE 9: Resolution of proposed DOA algorithm.

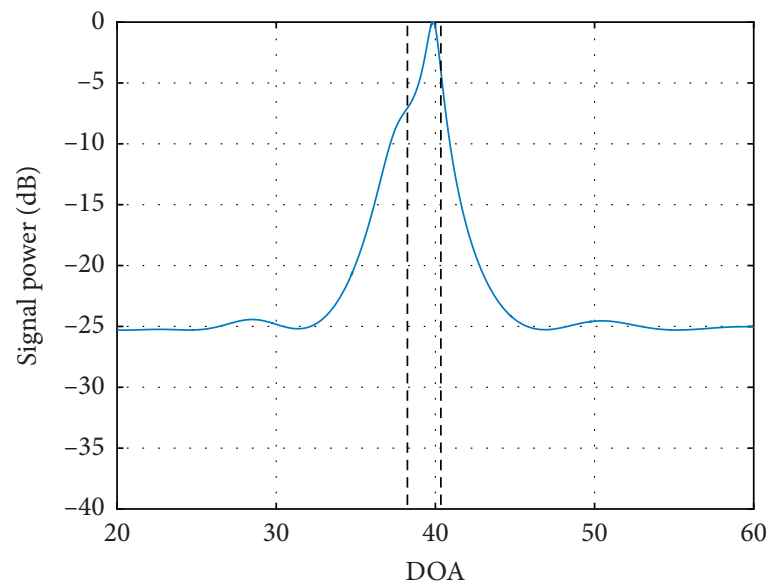

Algorithm proposed by Zhang et al. [18]

FIGURE 10: Resolution of DOA algorithm proposed by Zhang et al. [18]. 
literature [18] and 0.05 smaller than the algorithm in literature [15].

4.3. Experiment 3: Comparison of Resolution. The angles of incidence for the two signal sources tested in our current simulation are taken as $38.22^{\circ}$ and $40.32^{\circ}$, where $\mathrm{SNR}=-5 \mathrm{~dB}$ and the number of snapshots is 500 . As shown in Figure 9, when the SNR is low, the proposed method accurately resolves the direction of two nearby incoming waves. In contrast, as shown in Figure 10, the algorithm of Zhang et al. [18] does not satisfactorily resolve the direction of two nearby incoming waves.

\section{Conclusions}

We propose herein a method for interpolating a small number of antennas in the position of the coprime array to fill the holes of the virtual array derived from the coprime array. This method allows us to maintain the maximum degrees of freedom of the coprime array despite the unavailable holes in the virtual array. Meanwhile, the added antennas allow all holes of virtual arrays to be filled without expanding the original aperture of the coprime array, leading to the maximum number of degrees of freedom. In addition, we use the ESPRIT algorithm to estimate the DOA. Finally, comparing the proposed algorithm with simulation results demonstrates that the proposed algorithm increases the number of degrees of freedom of the coprime array and identifies more DOAs. Thus, the proposed algorithm substantially improves the resolution and accuracy of DOA estimates.

\section{Data Availability}

The data used to support the findings of this study are available from the corresponding author upon reasonable request and with permission from the funders.

\section{Conflicts of Interest}

The authors declare that there are no conflicts of interest regarding the publication of this paper.

\section{Acknowledgments}

This work was supported by the National Natural Science Foundation of China (Grant nos. 61901409 and 61661030).

\section{References}

[1] F. Wen, Z. Zhang, K. Wang, G. Sheng, and G. Zhang, "Angle estimation and mutual coupling self-calibration for ULAbased bistatic MIMO radar," Signal Processing, vol. 144, pp. 61-67, 2018.

[2] X. Wu, W. Zhu, and J. Yan, "A high-resolution DOA estimation method with a family of nonconvex penalties," IEEE Transactions on Vehicular Technology, vol. 67, no. 6, pp. 4493-4925, 2018.

[3] X. Zhang, "Direction of departure (DOD) and direction of arrival (DOA) estimation in MIMO radar with reduced- dimension MUSIC," IEEE Communications Letters, vol. 14, no. 12, pp. 1161-1163, 2010.

[4] Y. Gu and A. Leshem, "Robust adaptive beam-forming based on Interference covariance matrix reconstruction and steering vector estimation," IEEE Transactions on Signal Processing, vol. 60, no. 7, pp. 3881-3885, 2012.

[5] F. Sun, Q. Wu, Y. Sun, G. Ding, and P. Lan, "An iterative approach for sparse direction-of-arrival estimation in coprime arrays with off-grid targets," Digital Signal Processing, vol. 61, no. 3, pp. 35-42, 2017.

[6] S. Qin, Y. D. Zhang, and M. G. Amin, "Generalized coprime array configurations for direction-of-arrival estimation," IEEE Transactions on Signal Processing, vol. 63, no. 6, pp. 13771390, 2015.

[7] F. Wen and J. Shi, "Fast direction finding for bistatic EMVSMIMO radar without pairing," Signal Processing, vol. 173, Article ID 107512, 2020.

[8] Z. Wang and X. Zhang, "Extended coprime array configuration generating large-scale antenna co-array in massive MIMO system," IEEE Transactions on Vehicular Technology, vol. 68, no. 8, pp. 7841-7853, 2019.

[9] P. Pal and P. Vaidyanathan, "On application of LASSO for sparse support recovery with imperfect correlation awareness," in Proceedings of the 2012 Conference Record of the Forty Sixth Asilomar Conference on Signals, Systems and Computers (ASILOMAR), pp. 958-962, Pacific Grove, CA, USA, November 2012.

[10] P. Pal and P. P. Vaidyanathan, "Nested arrays: a novel approach to array processing with enhanced degrees of freedom," IEEE Transactions on Signal Processing, vol. 58, no. 8, pp. 4167-4181, 2010.

[11] P. P. Vaidyanathan and P. Pal, "Sparse sensing with co-prime samplers and arrays," IEEE Transactions on Signal Processing, vol. 59, no. 2, pp. 573-586, 2011.

[12] P. Pal and P. P. Vaidyanathan, "Coprime sampling and the music algorithm," in Proceedings of the 2011 Digital Signal Processing and Signal Processing Education Meeting (DSP/ SPE), pp. 289-294, Sedona, AZ, USA, January 2011.

[13] Z. Weng and P. M. Djurić, "A search-free DOA estimation algorithm for coprime arrays," Digital Signal Processing, vol. 24, no. 1, pp. 27-33, 2014.

[14] Y. D. Zhang, S. Qin, and M. G. Amin, "DOA estimation exploiting co-prime arrays with sparse sensor spacing," in Proceedings of the 2014 IEEE International Conference on Acoustics, Speech and Signal Processing (ICASSP), pp. 22672271, Florence, Italy, May 2014.

[15] C. Zhou and J. Zhou, "Direction-of-arrival estimation with coarray ESPRIT for coprime array," Sensors, vol. 17, no. 8, p. 1779, 2017.

[16] Y. d. Zhang, M. G. Amin, and B. Himed, "Sparsity-based DOA Estimation Using co-prime arrays," in Proceedings of the 2013 IEEE International Conference on Acoustics, Speech and Signal Processing, pp. 3967-3971, Vancouver, Canada, May 2013.

[17] E. Boudaher, Y. Jia, F. Ahmad, and M. G. Amin, "Multifrequency co-prime arrays for high-resolution direction-ofarrival estimation," IEEE Transactions on Signal Processing, vol. 63, no. 14, pp. 3797-3808, 2015.

[18] Y. Zhang, H. Xu, B. Ba et al., "Direction-of-arrival estimation for multiple sources based on the reconstruction of the coprime array," Acta Electronica Sinica, vol. 46, no. 12, pp. 109-115, 2018.

[19] C.-L. Liu and P. P. Vaidyanathan, "Remarks on the spatial smoothing step in coarray MUSIC," IEEE Signal Processing Letters, vol. 22, no. 9, pp. 1438-1442, 2015. 